\title{
Von Neumann e a Teoria de Álgebra de Operadores
}

RUY EXEL

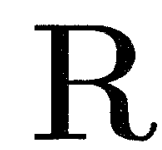

ESPONDENDO A UM QUESTIONÁRIO elaborado pela National Academy of Sciences dos EUA, von Neumann considerou seu trabalho em teoria de operadores como uma de suas três contribuições científicas mais importantes. Este trabalho, iniciado no final da década de 20 , tem origem no seu interesse em obter uma formalização matematicamente precisa para diversas teorias emergentes na época, dentre as quais se destaca a mecânica quântica.

No que veio a constituir aproximadamente um terço de sua extensa lista de publicações, von Neumann desenvolveu sua teoria de operadores no espaço de Hilbert, criando assim, não apenas uma nova teoria matemática, que comporta aplicaçôes à teoria de representaçôes de grupos infinitos e teoria ergódica, mas uma nova forma de pensamento, que permite modelar a lógica fugaz da mecânica quântica e que, com os avanços subseqüentes, proporcionou uma abrangente unificação de diversos campos da Matemática. As portas abertas pelo seu trabalho continuam a provocar desenvolvimentos cruciais na fronteira da matemática dos dias de hoje.

Von Neumann rapidamente reconheceu que o estudo de operadores em espaços de Hilbert requeria um ponto de vista mais amplo, no qual vários operadores deveriam ser estudados em conjunto, e que as relações algébricas entre estes mereceriam, talvez, mais atenção que o estudo das características que cada operador apresentava isoladamente (entretanto nâo se deve ignorar a importância de seu trabalho no estudo de single operator theory, sendo von Neumann um dos criadores do importantíssimo teorema espectral para operadores ilimitados).

Em 1929, no artigo Zur Algebra der Funktionaloperatoren und Theorie der normalen Operatoren, von Neumann (1929:370-427) iniciou seu estudo da teoria dos anéis de operadores, que hoje em dia, muito propriamente, são chamados de Álgebras de von Neumann. O conceito formal de anel, que surgiu pela primeira vez em 1921 com Emmy Noether, em 
artigo no Mathematische Annalen, foi imediatamente reconhecido como uma criação de fundamental importância na formulação da matemática moderna e, com von Neumann, ganhou alguns de seus mais importantes exemplos.

As teorias axiomáticas em Matemática muitas vezes permitem que se obtenha, de forma relativamente simples, uma classificação completa dos objetos de estudo, a exemplo da teoria dos espaços vetoriais: um espaço vetorial fica inteiramente determinado, uma vez que sua dimensão seja conhecida. Por outro lado, outraśs teorias tratam de objetos tão gerais, que uma classificação completa é reconhecidamente impossível. São raras as vezes em que um sistema de axiomas define um conjunto de objetos matemáticos que sejam sofisticados e gerais o suficiente para fazer, de seu estudo, um frutífero desafio intelectual, ao mesmo tempo que demarquem um terreno definido o suficiente para suscitar a possibilidade de classificação de todos os seus modelos possíveis. A teoria dos grupos de Lie vem à mente como um exemplo significativo. A análise cuidadosa da conexão misteriosa entre as ciências naturais e a Matemática é, freqüentemente, o melhor meio para que o intelecto humano possa vir a formular tais teorias. A teoria das álgebras de von Neumann é um exem-. plo importante deste método de criação intelectual, sendo a mecânica quântica seu ponto de conexão com o universo físico.

Uma álgebra de von Neumann, tecnicamente falando, é uma coleção $\mathcal{M}$ de operadores limitados no espaço de Hilbert, aqui denotado por $\mathcal{H}$, que, em primeiro lugar, forma uma subálgebra da álgebra $\mathcal{L}(\mathcal{H})$ de todos os operadores limitados em $\mathcal{H}$. Em segundo lugar, requer-se que $\mathcal{M}$ seja autoadjunta, ou seja, que contenha o adjunto de cada um dos seus elementos e, finalmente, que $\mathcal{M}$ seja fechada na topologia forte, isto é, na topologia da convergência pontual de operadores.

De fundamental importância para os estágios iniciais do desenvolvimento da teoria das álgebras de von Neumann, é a série de quatro artigos intitulados On Rings of Operators (1936: 116-229; 1937: 208$248 ; 1940: 96-161 ; 1943: 716-808)$, em três dos quais von Neumann contou com a importante contribuição de F. J. Murray.

Uma descrição da teoria das álgebras de von Neumann não pode omitir o resultado central de Zur Algebra der Funktionaloperatoren und Theorie der normalen Operatoren, que é o teorema do duplo comutante. Dada uma subálgebra auto-adjuntả $\mathcal{A}$ de $\mathcal{L}(\mathcal{H})$, que contenha o operador identidade, define-se o seu comutante como sendo o conjunto $\mathcal{A}^{\prime}$ dos ope- 
radores em $\mathcal{H}$ que comutam com cada um dos membros de $\mathcal{A}$. O teorema do duplo comutante afirma que o duplo comutante $\mathcal{A}^{\prime \prime}$ coincide com o fecho forte de $\mathcal{A}$. Além de importantes aplicações técnicas, este resultado simboliza uma das características estéticas mais marcantes da teoria, que é a harmonia entre seus aspectos algébricos e topológicos.

Uma álgebra de von Neumann $\mathcal{M}$ é chamada um fator quando seu centro consiste apenas dos múltiplos escalares da unidade, condição equivalente à inexistência de ideais fortemente fechados em $\mathcal{M}$. Para se ter idéia de como se relacionam estas duas condições note que, pelo teorema do duplo comutante, se vê imediatamente que as projeções espectrais de um operador auto-adjunto pertencente a $\mathcal{M}$, também pertencem a $\mathcal{M}$. Sendo assim, se $T$ é um operador pertencente ao centro de $\mathcal{M}$, qualquer uma de suas projeções espectrais $p$ será uma projeção central e, em conseqüência, $p \mathcal{M}$ será um ideal de $\mathcal{M}$. A inexistência de ideais acarreta, portanto, que os únicos operadores no centro de $\mathcal{M}$ devem ser os múltiplos da identidade. $\mathrm{O}$ exemplo básico de fator é a álgebra $\mathcal{L}(\mathcal{H})$ mencionada. No caso em que $\mathcal{H}$ tem dimensão finita, $\mathcal{L}(\mathcal{H})$ reduz-se à álgebra $M_{n}(\mathbf{C})$, das matrizes complexas $n \times n$, onde $n$ é a dimensão de $\mathcal{H}$.

Embora apenas em artigo de 1949 (401-485) von Neumann tenha provado o teorema de decomposição em fatores, a relevância dos fatores foi imediatamente reconhecida. Os fatores são os constituintes fundamentais da teoria e a compreensão destes afeta o conhecimento de todos os outros exemplos. A grosso modo, o teorema de decomposição reza que toda álgebra de von Neumann pode ser reduzida, relativamente ao seu centro, a uma integral direta de fatores. $O$ conceito de integral direta corresponde a uma generalização de somas diretas, para o caso em que o conjunto de fatores é parametrizado, não por um conjunto discreto, mas por um espaço de medida contínuo.

Com o teorema de decomposição, a compreensão da estrutura das álgebras de von Neumann se reduz à classificação dos fatores, e a preocupação em se chegar a este objetivo é perfeitamente visível na série Rings of Operators. Ali encontramos os primeiros resultados importantes. Para se obter uma primeira classificação dos fatores, Murray e von Neumann desenvolveram a teoria de comparação de projeções. Uma projeção é um elemento $p$ de uma álgebra de von Neumann que satisfaz $p^{2}=p^{*}=p$. Estas relações acarretam que $p$ é uma projeção ortogonal, no sentido usual, sobre um subespaço do espaço de Hilbert subjacente.

Dadas duas projeções $p$ e $q$ numa álgebra de von Neumann $\mathcal{M}$, diz- 
se que $p$ e $q$ são equivalentes, em símbolos $p \sim q$, se existe um operador $u$ em $\mathcal{M}$ tal que $p=u u^{*}$ e $q=u^{*} u$. Com esta noção de equivalência, Murray e von Neumann introduziram uma ordem parcial no conjunto das projeções em $\mathcal{M}$. Isto é, diz-se que $p \preceq q$ se $p$ é equivalente a uma subprojeção de $q$, ou seja, a uma projeção cuja imagem está contida na imagem de $q$. De importância central é o fato de que esta ordem é total num fator, isto é, ou bem $p \preceq q$ ou então $q \preceq p$. Uma projeção $p$ é chamada infinita se $p$ é equivalente a uma sua subprojeção e, em caso contrário, $p$ e dita finita. Se a identidade da álgebra de von Neumann $\mathcal{M}$, que é sempre uma projeção, for finita, então a álgebra é também chamada finita, e similarmente se definem álgebras infinitas.

Relacionada à teoria de comparação de projeções, Murray e von Neumann introduziram a sua funçấo dimensão. Mais precisamente, foi demonstrado que, para um fator $\mathcal{M}$, existe uma função $D$, definida no conjunto das projeções de $\mathcal{M}$, e tomando valores reais positivos, incluindo o valor infinito, tal que

(i) $D(p)>0$ se $p \neq 0$,

(ii) $D(p)=D(q)$ se e somente se $p \sim q$,

(iii) $D(p) \leq D(q)$ se e somente se $p \preceq q$ e

(iv) $D(p+q)=D(p)+D(q)$ se $p$ e $q$ são projeções ortogonais, isto é $p q=0$.

Isto reproduz a noção clássica de dimensão de projeções num espaço de Hilbert e indica a possibilidade de se obter modelos não clássicos em física quântica. A demonstração da existência e unicidade de $D$ segue idéias relacionadas com o conceito de medida de Haar em grupos localmente compactos, um campo da matemática que também conta com importantes contribuições de von Neumann (1934:106-114), responsável pela demonstração da unicidade da medida de Haar para grupos compactos.

A natureza do conjunto de valores da função dimensão estabelece a primeira classificação dos fatores. Este conjunto pode ser:

(i) $\{0,1, \ldots, n\}$, onde $n$ é um inteiro, incluindo infinito.

(ii) $[0,1]$, isto é, o intervalo real fechado de 0 a 1 , 
(iii) $[0, \infty]$,

(iv) $\{0, \infty\}$.

$\mathrm{Na}$ primeira situação o fator se denomina de tipo $I_{n}$ e, neste caso, é necessariamente isomorfo a $M_{n}(\mathbf{C})$ ou, no caso em que $n$ é infinito, a $\mathcal{L}(\mathcal{H})$. O segundo caso corresponde ao fato de que a identidade é finita, não existindo projeções minimais, e o fator é denominado de tipo $I I_{1}$. A alternativa seguinte define os fatores de tipo $I I_{\infty}$. Dentre todos, os mais misteriosos, ao menos na época, são os fatores de tipo $I I I$, que correspondem ao último caso.

A existência de fatores do tipo $I I$ e $I I I$, embora prevista pela teoria, não era, de maneira alguma, óbvia. Para a construção de exemplos Murray e von Neumann lançaram mão de elementos da teoria ergódica. É interessante notar que a própria teoria ergódica, que estava nascendo na época, contou também com importantes contribuições de von Neumann. Os primeiros exemplos de fatores de tipo $I I$ e $I I I$ foram obtidos da seguinte maneira. Dado um espaço de medida $(\Omega, m)$ com uma ação de um grupo discreto enumerável $G$, considere a álgebra constituída por todas as funçôes mensuráveis e essencialmente limitadas em $\Omega$, denotada por $L^{\infty}(\Omega)$. Cada elemento $g$ do grupo, define um automorfismo de $L^{\infty}(\Omega)$ através da fórmula $g \cdot f(\omega)=f\left(g^{-1} \omega\right)$. Uma construção tęcnicamente complexa permite que se obtenha uma álgebra de von Neumann $\mathcal{M}$, que contenha, de uma maneira natural, uma cópia de $L^{\infty}(\Omega)$, assim como uma família de elementos unitários $\left\{u_{g}\right\}_{g \in G}$, satisfazendo

$$
u_{g} f u_{g}^{*}=g \cdot f .
$$

A condição para que tal álgebra seja um fator é a ação ser livre e ergódica. Neste contexto, livre significa que a medida do conjunto $\{\omega: g \omega=\omega\}$ é zero para todo elemento $g$ não-trivial, enquanto a definição de ergodicidade requer que os únicos subconjuntos $S$ de $\Omega$ tais que a medida de $g(S)-S$ seja zero para todo elemento $g$ do grupo, sejam aqueles que têm medida zero ou cujo complementar tenha medida zero.

A construção de uma álgebra de operadores a partir de uma ação de grupo num espaço de medida é uma importante idéia de Koopman, que foi o orientador de Murray. Esta construção inaugurou uma frutífera conexão entre duas teorias aparentemente não relacionadas, a saber, a teoria dos anéis de operadores e a teoria dos sistemas dinâmicos. Tal conexão sobrevive até os dias de hoje com pleno vigor, sendo que alguns resultados cruciais neste assunto foram obtidos muito recentemente 
por Giordano, Putnam e Skau (1983), fornecendo uma classificação de homeomorfismos minimais no conjunto de Cantor.

Voltando ao problema de se construir fatores de tipo $I I$ e $I I I$, Murray e von Neumann estudaram, inicialmente, o caso em que $\Omega$ admite uma medida invariante. Neste caso, se existem átomos em $\Omega$, isto é, subconjuntos indecomponíveis de medida positiva, o fator resultante será de tipo $I$ - finito ou infinito conforme a medida de $\Omega$ seja finita ou infinita. $\mathrm{Na}$ ausência de átomos, $\mathcal{M}$ será de tipo $I I$, finito ou infinito como mencionado.

No terceiro artigo da série Rings of Operators, von Neumann logrou encontrar os primeiros casos de fatores de tipo $I I I$. Os exemplos obtidos resultaram também de sistemas ergódicos que, desta vez, não admitissem medida invariante alguma.

Exemplos concretos destas situações são:

- a ação do grupo dos inteiros no círculo, por meio de rotações de ângulo irracional, resultando num fator de tipo $I I_{\mathbf{1}}$,

- a ação do grupo dos números racionais na reta real, por translação, resultando num fator de tipo $I I_{\infty} \mathrm{e}$

- a ação, na reta real, do grupo de transformações afins $x \mapsto a x+b$, onde $a$ e $b$ são números racionais, resultando num fator do tipo $I I I$.

Ainda hoje não temos uma classificação completa de todos os fatores. Entretanto, alguns dos desenvolvimentos mais marcantes na teoria de álgebras de von Neumann desde então, têm sido provocados pelo desejo de se refinar a classificação em tipos originada por Murray e von Neumann. Dentre estes destaca-se o trabalho de Connes (1973:133-252), cuja classificação dos fatores de tipo $I I I$ lhe valeu a medalha Fields. Connes, utilizando a teoria de Tomita-Takesaki, provou que os fatores do tipo III se dividem em classes $I I I_{\lambda}$, onde $\lambda$ é um parâmetro real, relacionado com o espectro de um grupo de automorfismos da álgebra que está codificado na sua própria estrutura algébrica.

Em On Ring of Operators II e IV Murray e von Neumann dedicaram esforço considerável ao estudo do problema de isomorfismo espacial de fatores. Dados dois fatores isomorfos entre si como álgebras abstratas, isto é, sem consideração ao espaço de Hilbert subjacente, pergunta-se sob que condições este isomorfismo é espacial; ou seja se existe um operador unitário $u$ entre os respectivos espaços de Hilbert, de tal forma 
que o isomorfismo dado coincida com a conjugação por $u$. No primeiro (1937:208-248) mostraram que no caso de fatores finitos, a questão crucial reside na análise da constante de acoplamento (coupling constant).

Para ser mais preciso, dado um fator $\mathcal{M}$ operando num espaço $\mathcal{H}$, seja $\mathcal{M}^{\prime}$ o seu comutante. Dado um vetor não nulo $x$ qualquer em $\mathcal{H}$, considere o espaço $[\mathcal{M} x]$, que é claramente invariante por $\mathcal{M}$. Disto seguese que a correspondente projeção ortogonal comuta $\operatorname{com} \mathcal{M}$ e, portanto, é um elemento de $\mathcal{M}^{\prime}$. Da mesma forma a projeção ortogonal em $\left[\mathcal{M}^{\prime} x\right]$ pertence a $\mathcal{M}$. Sendo assim, a constante de acoplamento é definida como sendo o quociente

$$
D\left(\left[\mathcal{M}^{\prime} x\right]\right) / D^{\prime}([\mathcal{M} x]),
$$

onde $D$ e $D^{\prime}$ são as funções dimensão de $\mathcal{M}$ e $\mathcal{M}^{\prime}$, respectivamente. Um ponto importante a ser ressaltado é que esta constante não depende da escolha de $x$, sendo conseqüentemente um invariante intrínsico da álgebra $\mathcal{M}$ e de sua posição relativa ao seu comutante.

A solução do problema do isomorfismo espacial obtida por Murray e von Neumann afirma que dois fatores finitos, isomorfos entre si, são unitariamente equivalentes se, e somente se, as respectivas constantes de acoplamento coincidem. Murray e von Neumann estenderam estes resultados, exceto para o caso em que $\mathcal{M}$ é de tipo $I I I$ e o caso em que $\mathcal{M}$ é de tipo $I I_{\infty}$ e $\mathcal{M}^{\prime}$ é de tipo $I I_{1}$. Estes casos vieram a ser estudados e resolvidos mais tarde por Griffin, Dye e Kadison.

Um resultado de extrema elegância foi obtido em 1943 (716-808), esclarecendo a relação entre fatores de tipo $I I_{\infty}$ e $I I_{1}$. Dado um fator $\mathcal{M}$ do tipo $I I_{\infty}$, sabe-se que a identidade é uma projeção infinita e, portanto pode ser escrita como a soma de uma família ortogonal infinita $\left\{p_{i}\right\}$ de projeções finitas, mutuamente equivalentes. Considerando a equivalência entre $p_{i}$ e $p_{j}$, existirá, portanto, uma isometria parcial $u_{i j}$ em $\mathcal{M}$ tal que $u_{i, j}^{*} u_{i, j}=p_{j}$ e $u_{i, j} u_{i, j}^{*}=p_{i}$. Desta forma, o conjunto $\left\{u_{i j}\right\}$ passa a ser uma família de unidades matriciais em $\mathcal{M}$, gerando, portanto, um fator de tipo $I_{\infty}$. Por outro lado, a subálgebra $p_{0} \mathcal{M} p_{0}$ será um fator de tipo $I I_{1}$, uma vez que $p_{0}$ é uma projeção finita. Um raciocínio simples conduz à conclusão de que $\mathcal{M}$ é nada mais que o produto tensorial de um fator de tipo $I I_{1}$ por um de tipo $I_{\infty}$. Dito de outra forma, todo fator de tipo $I I_{\infty}$ pode ser visto como a álgebra das matrizes infinitas sobre um fator de tipo $I I_{1}$.

A classificação de fatores em tipos $I_{n}, I I_{1}, I I_{\infty}$ e $I I I$, aliada à prova da existência de fatores de cada um dos tipos previstos, levantava 
a questão crucial quanto a esta classificação ser completa, ou seja, se dois fatores do mesmo tipo deveriam ser, necessariamente, isomorfos. Resolvendo esta questão, Murray e von Neumann obtiveram exemplos não isomorfos de fatores do tipo $I I_{1}$, utilizando, para istọ, a teoria de representação de grupos. Dado um grupo discreto $G$, considere o espaço de Hilbert $l_{2}(G)$, constituído por todas as seqüências $\left(\lambda_{g}\right)_{g \in G}$ de números complexos, indexadas por $G$, satisfazendo

$$
\sum_{g \in G}\left|\lambda_{g}\right|^{2}<\infty .
$$

A representação regular de $G$ em $l_{2}(G)$ consiste em se associar, para cada elemento $g$ de $G$, um operador unitário $u_{g}$ em $l_{2}(G)$, através da fórmula

$$
u_{g}(\lambda)_{h}=\lambda_{g^{-1} h} .
$$

A álgebra de von Neumann gerada pela imagem da representação regular resulta num fator se, e somente se, a classe de conjugação de todo elemento não-trivial de $G$ for infinita. Esta propriedade caracteriza os grupos chamados ICC (infinite conjugacy class). Neste caso é fácil provar que tal fator é de tipo $I I_{1}$. É o caso, por exemplo, do grupo livre com $n$ geradores, denotado por $F_{n}$, e também do grupo $S_{\infty}$ constituído por todas as permutações de um conjunto infinito enumerável, que movam apenas um subconjunto finito.

Com o objetivo de distinguir a classe de isomorfismo entre estas álgebras, foi definida uma propriedade de comutatividade fraca para álgebras de von Neumann, a qual é verificada para o caso de $S_{\infty}$, embora falhe para $F_{2}$, mostrando assim que existem fatores de tipo $I I_{1}$ não isomorfos entre si. A propósito, um dos grandes problemas não resolvidos até hoje, e que tem sido objeto de intensa investigação, é a questão do isomorfismo entre os fatores associados a grupos livres com um número distinto de geradores. Esta questão levou Voiculescu et al. (1992) a introduzirem sua teoria de probablidade não comutativa, teoria esta que tem um interesse intrínseco significativo.

O caso do grupo $S_{\infty}$ chamou a atenção para uma propriedade extremamente importante. Uma álgebra de von Neumann $\mathcal{M}$ é chamada hiperfinita (a nomenclatura original dada por von Neumann foi aproximadamente finita) se existe uma cadeia crescente de subálgebras de dimensão finita, cuja reunião é fortemente densa em $\mathcal{M}$. 
Num dos sucessos mais significativos atingidos pela teoria de classificação de fatores, Murray e von Neumann provaram que dois fatores hiperfinitos de tipo $I I_{1}$ são necessariamente isomorfos. A demonstração deste fato consiste de uma longa e sofisticada série de argumentos. Em 1976, Alain Connes (1973:133-252) obteve uma importante generalização deste resultado para fatores mais gerais.

Já foi dito que a motivação de von Neumann, no seu estudo de operadores, foi entre outras, a tentativa de estabelecimento de uma base formal para a mecânica quântica. Entretanto, os observáveis físicos são freqüentemente ilimitados, o que faz com que os operadores ilimitados sejam objeto de grande interesse. Por outro lado, a teoria de álgebras de von Neumann trata de operadores limitados, já que, em caso contrário, as propriedades algébricas tornam-se de difícil compreensão. Por exemplo, dados operadores ilimitados $T_{1}$ é $T_{2}$, não é, de forma alguma óbvia, como obter a soma $T_{1}+T_{2}$. O problema que se coloca é que os domínios de $T_{1}$ e $T_{2}$ podem ter interseç̧ão vazia. Entretanto, em física quântica, manipulações formais com operadores ilimitados são rotineiramente executadas e, embora o significado matemático não seja sempre absolutamente cristalino, a utilidade destes cálculos é inegável. Com sua descoberta de fatores de tipo $I I_{1}$, Murray e von Neumann obtiveram um sucesso importante no sentido de atribuir significado matemático para cálculos com operadores ilimitados. Para se compreender tal fenômeno, é preciso introduzir o conceito de operadores afiliados a uma álgebra de von Neumann. A saber, um operador ilimitado, densamente definido e fechado $T$ é dito afiliado a uma álgebra $\mathcal{M}$, se $T$ comuta com todo operador em $\mathcal{M}^{\prime}$. Murray e von Neumann (1936) provaram que, para tais operadores, a maioria das propriedades desejáveis para o cálculo de operadores é satisfeita. Por exemplo, tais operadores podem ser somados e multiplicados entre si, formando uma álgebra no sentido usual da palavra.

Existe ainda enorme quantidade de contribuições significativas de von Neumann à teoria de operadores que não foram sequer citadas, como, por exemplo, álgebras abelianas maximais, teoria não comutativa de integração, antiautomorfismos e produtos diretos infinitos.

Como já enfatizado, sua obra abriu um campo profundamente fértil em matemática que, ainda hoje, é objeto de alguns dos mais fascinantes desenvolvimentos da ciência. A sua extensão e o seu alcance fazem com que seja completamente impossivel descrever, ainda que superficialmente, em uma única palestra, todos esses desenvolvimentos. Entretanto, eu gostaria de comentar, com algum detalhe, a teoria de Vaughan Jones 
sobre fatores do tipo $I I_{1}$ por duas razões. Primeiro, por sua magnífica elegância e, em segundo lugar, pelo fato de ter trazido para o universo de aplicações das álgebras de von Neumann, uma nova área da matemática: a teoria de nós.

A teoria de nós trata, como o nome indica, de se classificar, matematicamente, as diversas possibilidades de atar um ou mais pedaços de barbante, formando um nó. Além de seu interesse intrínseco, a teoria de nós encontra aplicações em topologia, geometria diferencial, teoria das singularidades, mecânica estatística e em genética, na qual o polinômio de Jones é usado para se estudar cadeias de DNA.

As aplicações do estudo de álgebras de von Neumann, encontradas por Jones em teoria de nós, foram obtidas de maneira totalmente não-intencional, e resultaram do interesse de Jones em desenvolver uma Teoria de Galois para fatores do tipo $I I_{1}$. Neste estudo, Jones deparouse com determinadas relações algébricas que permitiram a introdução de um invariante polinomial para nós, ou seja, um método de se associar um polinômio a um nó, através do qual, freqüentemente, pode-se distinguir entre dois nós não equivalentes. Pelo seu trabalho nesta área, Jones fez jus à segunda medalha Fields atribuída a um cientista dedicado ao estudo de álgebras de von Neumann.

O ponta-pé inicial para a teoria de Jones é a noção de constante de acoplamento, introduzida por Murray e von Neumann, conforme já mencionado. No caso de um fator $\mathcal{M}$, do tipo $I I_{1}$, Murray e von Neumann demonstraram que existe uma função linear $\operatorname{tr}$, a valores complexos, definida para todos os elementos de $\mathcal{M}$, que obedece às relações:

(i) $\operatorname{tr}(a b)=\operatorname{tr}(b a)$ para quaisquer elementos $a$ e $b$ em $\mathcal{M}$,

(ii) $\operatorname{tr}\left(a^{*} a\right) \geq 0$ para todo $a$ em $\mathcal{M ~ e}$

(iii) $\operatorname{tr}(p)=D(p)$ para toda projeção $p$ em $\mathcal{M}$.

A esta função dá-se o nome de traço, devido às semelhanças com as propriedades de traço de matrizes complexas. Utilizando-se tr, define-se o espaço $L_{2}(\mathcal{M})$ como sendo o espaço de Hilbert obtido a partir de $\mathcal{M}$, com o produto escalar

$$
\langle a, b\rangle=\operatorname{tr}\left(b^{*} a\right) .
$$

A cada elemento $a$ de $\mathcal{M}$, define-se um operador $T_{a}$ em $L_{2}(\mathcal{M})$, pela fórmula $T_{a}(b)=a b$. Isto define o que se chama de uma representação 
de $\mathcal{M}$ em $L_{2}(\mathcal{M})$, através da qual podemos interpretar $\mathcal{M}$ como uma álgebra de operadores em $L_{2}(\mathcal{M})$. Esta representação é chamada de forma standard de $\mathcal{M}$.

Conforme o espírito da teoria de Galois, Jones estava interessado no estudo de subfatores $\mathcal{N} \subseteq \mathcal{M}$. Como as propriedades que distinguem fatores do tipo $I I_{1}$ serão necessariamente herdadas por $\mathcal{N}$, este será também de tipo $I I_{1}$.

O substituto para o conceito de índice para extensões de corpos da teoria de Galois é o índice de Jones $[\mathcal{M}, \mathcal{N}]$, definido como sendo a constante de acoplamento para $\mathcal{N}$, vista como uma álgebra de operadores em $L_{2}(\mathcal{M})$.

O primeiro indicador de que algo verdadeiramente fantástico se oculta nesta teoria é o teorema demonstrado por Jones segundo o qual os possíveis valores para o índice são

- qualquer número real $r \geq 4$.

- qualquer número da forma $r=4 \cos ^{2} \pi / n$, onde $n$ é um inteiro maior ou igual a três.

Consideremos, a partir de agora, uma inclusão $\mathcal{N} \subseteq \mathcal{M}$ de fatores do tipo $I I_{1}$. A chamada construção básica, devida a Cristensen e Skau, é um ingrediente importante na obtenção do invariante polinomial de Jones. Esta construção está baseada no fato que existe uma esperança condicional, ou seja, uma aplicação $E: \mathcal{M} \rightarrow \mathcal{N}$ que satisfaz:

(i) $E(a x b)=a E(x) b$ se $a, b \in \mathcal{N}$ e $x \in \mathcal{M}$.

(ii) $\operatorname{tr}(a E(x))=\operatorname{tr}(a x)$ para $a \in \mathcal{N}$ e $x \in \mathcal{M}$.

(iii) $E\left(x^{*}\right)=E(x)^{*}$ para $x \in \mathcal{M}$.

Além disso, é possível demonstrar-se que o operador $E$ pode ser estendido a um operador em $L_{2}(\mathcal{M})$, extensão esta que será, doravante, denotada por $e$. Sendo assim, pode-se obter uma álgebra maior que $\mathcal{M}$, construindo-se a álgebra $\langle\mathcal{M}, e\rangle$, gerada por $\mathcal{M} \cup\{e\}$. Jones provou que se o índice de $\mathcal{N}$ em $\mathcal{M}$ é finito, então $\langle\mathcal{M}, e\rangle$ é também um fator do tipo $I I_{1}$ e que o índice se preserva no sentido que

$$
[\langle\mathcal{M}, e\rangle, \mathcal{M}]=[\mathcal{M}, \mathcal{N}] .
$$


A construção básica, mencionada, consiste, precisamente, em se produzir a álgebra $\langle\mathcal{M}, e\rangle$ a partir do $\operatorname{par} \mathcal{N} \subseteq \mathcal{M}$. É interessante que, uma vez aplicada esta construção, encontramo-nos na posição de repetila. Portanto, esse procedimento pode ser feito uma quantidade infinita de vezes. Obtém-se, assim, uma seqüência $\mathcal{M}_{i}$ de fatores do tipo $I I_{1}$, onde $\mathcal{M}_{i+1}=\left\langle\mathcal{M}_{\imath}, e_{i}\right\rangle$. As relações algébricas satisfeitas pela seqüência $\left\{e_{i}\right\}$ são de crucial importância. Sendo $\tau=[\mathcal{M}, \mathcal{N}]^{-1}$, tem-se:

(i) $e_{i}^{2}=e_{i}, e_{i}^{*}=e_{i}$,

(ii) $e_{\imath} e_{i \pm 1} e_{i}=\tau e_{i}$,

(iii) $e_{i} e_{3}=e_{j} e_{i}$ se $|i-j| \geq 2$,

(iv) $\operatorname{tr}\left(x e_{n+1}\right)=\tau \operatorname{tr}(x)$ quando $x$ é um elemento da álgebra gerada por $e_{1}, \ldots, e_{n}$.

Para prosseguir precisamos introduzir o importante conceito de braid. Um braid, ou seja, uma trança, consiste em se tomar duas varetas, cada uma das quais com $n$ orifícios, igualmente espaçados, colocadas horizontalmente, uma acima da outra a uma certa distância. Toma-se, também, $n$ cordões e ata-se uma das extremidades de cada cordão a um dos orifícios da vareta superior, sendo que e a outra extremidade é atada a um dos orifícios da vareta inferior. Assim, obtém-se uma trança que, naturalmente, pode assumir diversas configurações distintas. $O$ chamado braid group, ou o grupo das tranças, $B_{n}$ consiste do conjunto de todas estas configuraçôes possíveis. A estrutura de grupo de $B_{n}$ é dada pela seguinte operação: o produto de duas tranças é obtido fazendo-se coincidir a vareta inferior de uma destas com a superior da outra e, então, eliminando-se estas duas varetas, após atar-se os cordóes superiores com os inferiores, obedecendo o orifício em que cada cordão estava amarrado.

Entre as possíveis tranças, destacam-se $n-1$ elementos fundamentais $\left\{\sigma_{1}, \ldots, \sigma_{n-1}\right\}$. Começando-se com a trança trivial, isto é, aquela em que os cordões são amarrados verticalmente, tome o $i$-ésimo cordão juntamente com seu vizinho da direita, desamarre-os da vareta inferior, dê meia volta e torne e amarrá-los, trocando, assim, sua posição. Desta forma, obtém-se a trança $\sigma_{i}$, já mencionada. É um antigo resultado de Artin que estas tranças fundamentais satisfazem as relações:

(j) $\sigma_{i} \sigma_{\jmath}=\sigma_{j} \sigma_{\imath}$ se $|i-j| \geq 2$

(ii) $\sigma_{\imath} \sigma_{i+1} \sigma_{\imath}=\sigma_{i+1} \sigma_{i} \sigma_{i+1}$ 
e que, além disso, estas relações definem $B_{n}$ completamente, formando uma apresentação deste grupo.

A aparente semelhança entre estas relações e as relações entre os elementos $e_{i}$ foi notada por um membro da platéia durante uma conferência proferida por Vaughan Jones. Matematicamente, esta semelhança expressa-se pelo fato de que a associação

$$
\sigma_{i} \mapsto(t+1) e_{i}-1,
$$

estabelece uma representação de $B_{n}$ na álgebra de von Neumann na qual residem os $e_{i}$. Aqui, $t$ é dado pela equação $\tau=2+t+t^{-1}$, lembrando que $\tau=[\mathcal{M}, \mathcal{M}]$. Calculando-se o traço do elemento correspondente, obtém-se um número complexo que depende do valor do índice $[\mathcal{M}, \mathcal{M}]$ ou, mais explicitamente, do parâmetro $t$.

Ainda que, neste ponto, a teoria torne-se ligeiramente intrincada, não é preciso muita imaginação para perceber que tranças e nós têm uma íntima relação entre si. Mais precisamente, prova-se que todo nó pode ser obtido pelo fechamento de uma trança. Embora a trança que deve ser utilizada para se obter um dado nó não seja única, é um teorema de Markov que duas tranças que se fechem num mesmo nó podem ser obtidas uma da outra por uma seqüência de transformações chamada movimentos de Markov.

Para que o traço da representação de uma trança $\beta \in B_{n}$ dependesse apenas do nó que desta se obtém, Jones levou em conta o efeito dos movimentos de Markov no cálculo do traço e definiu

$$
V_{\beta}(t)=\left(-\frac{t+1}{\sqrt{t}}\right)^{n-1}(\sqrt{t})^{e(\beta)} \operatorname{tr}(\beta)
$$

onde $e(\beta)$ é a imagem de $\beta$ na abelianização $\mathbf{Z}$ de $B_{n}$. Assim, fica definida uma função de $t$ que é um invariante para o nó $\beta$, no sentido que dois nós equivalentes terão, necessariamente, a mesma função $V$. Mais importante que isto é o fato de dois nós distintos muitas vezes terem invariantes distintos, o que permite, de forma concreta e objetiva, perceber tal distinção. $\mathrm{Na}$ verdade, invariantes polinomiais para nós não são propriamente uma novidade, uma vez que o polinômio de Alexander foi introduzido em 1923. O aspecto de novidade do polinômio de Jones, entretanto, reside no fato de que certos nós não distinguidos pelo polinômio de Alexander, o são pelo polinômio de Jones. O leitor interessado encontrará mais informações sobre o trabalho de Jones em seus artigos publicados em 1976 e 1983. 
O autor gostaria de expressar seus agradecimentos a Severino Toscano de Melo e Michael Forger, que colaboraram na forma de uma leitura crítica da versão preliminar do presente trabalho.

Referências bibliográficas

CONNES, A. Une classification des facteurs de type III. Ann. Ecole Norm. Supp. 6 1973, p. 133-252.

- Classification of injective factors; cases $I I_{1}, I I_{\infty}, I I I_{\lambda}, \lambda \neq 1$. Ann. Math. 104 1976, p. 73-115.

GIORDANO, T.; PUTNAM, I. F. \& SKAU, C. Topological orbit equivalence and $C^{*}$ crossed products. Tech. rep., University of Trondheim, 1993.

JONES, V. Index for Subfactors. Invent. Math. 72 1983, p. 1-25.

Subfactors and Knots, v. 80 of Regional Conference Series in Mathematics. American Mathematical Society, 1991.

KADISON, R. V. Theory of operators. Part II. Operator algebras. Bull. Amer. Math. Soc. v. 64, Part II 1958, p. 61-85.

MURRAY, F. J. Theory of operators. Part I. Single operators. Bull. Amer. Math. Soc. v. 64, Part II 1958, p. 57-60.

MURRAY, F. J. \& VON NEUMANN, J. On rings of operators. Ann. of Math. 37 1936, p. 116-229.

MURRAY, F. J. \& VON NEUMANN, J. On rings of operators, II. Trans. Amer. Math. Soc. 41 1937, p. 208-248.

On rings of operators, IV. Ann. of Math. 44 1943, p. 716-808.

ULAM, S. John von Neumann. Bull. Amer. Math. Soc. v. 64, Part II 1958, p. 1-49.

VOICULESCU, D. V.; DYKEMA, K. \& NICA, A. Free random variables, v. 1 of CRM Monograph Series. American Mathematical Society, 1992.

VON NEUMANN, J. Zur Algebra der Funktionaloperatoren und Theorie der normalen Operatoren. Math. Ann. 102 1929, p. 370-427. 
VON NEUMANN, J. Zum Haarschen Maass in topologischen Gruppen. Compositio Math. 1 1934, p. 106-114.

On rings of operators, III. Ann. of Math. 411940, p. 96-161.

On rings of operators, Reduction theory. Ann. of Math. 50 1949, p. $401-485$.

Ruy Exel é professor do Departamento de Matemática do Instituto de Matemática e Estatística da USP.

Palestra feita pelo autor no encontro A obra e o legado de John von Neumann, organizado pelo Instituto de Estudos Avançados da USP e pela Academia Brasileira de Ciências no Instituto de Matemática e Estatística da USP em 14 de novembro de 1995. 